\title{
High level of homeobox A9 and PBX homeobox 3 expression in gastric cancer correlates with poor prognosis
}

\author{
YING-YU MA ${ }^{1,2}$, YUANCHENG ZHANG ${ }^{1}$, XIAO-ZHOU MOU ${ }^{2}$, \\ ZHENG-CHUANG LIU ${ }^{3}$, GUO-QING RU ${ }^{4}$ and ERGUANG LI ${ }^{1}$

\begin{abstract}
${ }^{1}$ Medical School and Jiangsu Laboratory of Molecular Medicine, Nanjing University, Nanjing, Jiangsu 210093; ${ }^{4}$ Department of Pathology, Zhejiang Provincial People's Hospital, Hangzhou, Zhejiang 310014, P.R. China
\end{abstract} \\ ${ }^{2}$ Clinical Research Institute, Zhejiang Provincial People's Hospital; ${ }^{3}$ Key Laboratory of Gastroenterology of Zhejiang;
}

Received February 2, 2016; Accepted February 28, 2017

DOI: $10.3892 / 01.2017 .6937$

\begin{abstract}
The homeobox protein homeobox (HOXA9) is a transcriptional factor that regulates patterning during embryogenesis and controls cell differentiation. HOXA9 dysfunction has been implicated in certain cancers. However, the role of HOXA9 in gastric cancer is poorly understood. The present study investigated HOXA9 and its cofactor PBX homeobox 3 (PBX3) expression in patients with gastric cancer. Paired tissue samples from 24 patients and paraffin embedded tissues of gastric cancer patients (104 males and 24 females) were included. HOXA9 and PBX3 expression levels were determined by reverse transcription quantitative polymerase chain reaction in fresh tissues, and by immunohistochemical staining in paraffin embedded tissues. The association between HOXA9/PBX3 expression and clinicopathological features was established. The results demonstrated that HOXA9 and PBX3 mRNA levels were significantly upregulated $(\mathrm{P}=0.032$ for HOXA9 and $\mathrm{P}=0.031$ for $\mathrm{PBX} 3)$ in gastric cancer tissue. Immunohistochemical staining revealed that HOXA9 expression was associated with differentiation, lymph node metastasis and tumor-node-metastasis (TNM) stage, and PBX3 expression was associated with lymph node metastasis and TNM stage. Correlation analysis revealed a high coincidental expression of HOXA9 and PBX3 levels in gastric cancer $(r=0.391 ; \mathrm{P}<0.001)$. Survival analysis showed that high expression of HOXA9 or PBX3 was associated with poor survival of gastric cancer, and multivariate analysis using Cox's regression model showed that PBX3 expression was an independent prognostic factor in gastric cancer. There was elevated expression of HOXA9 and PBX3 in gastric
\end{abstract}

Correspondence to: Mrs. Ying-Yu Ma or Professor Erguang Li, Medical School and Jiangsu Laboratory of Molecular Medicine, Nanjing University, 22 Hankou Road, Nanjing, Jiangsu 210093, P.R. China

E-mail: myy011525@163.com

E-mail: erguang@nju.edu.cn

Key words: homeobox A9, PBX homeobox 3, gastric cancer, progression, prognosis cancer patients, and high-level expression of those proteins was associated with poor prognosis of gastric cancer. The present study underlines the significance of HOXA9/PBX3 in the development of gastric cancer.

\section{Introduction}

Gastric cancer (GC) is a major public health issue, and is the second leading cause of cancer-associated mortality worldwide, particularly in East Asia (1). Current treatment modalities for GC include surgery, radiotherapy, chemotherapy and their combinations. New therapies, including molecule-targeted therapy, have been prescribed for gastric cancer due to their marked benefits in reducing disease recurrence and increasing long-term survival $(2,3)$. Tumor invasion and metastasis, which are primary causes for treatment failure or mortality among cancer patients, involve multiple steps. The process involves regulation at the molecular level of adhesive molecules, proteolytic enzymes and cell growth and angiogenesis factors, and its mechanism is not yet fully understood (4). Therefore, searching for tumor-specific biomarkers for invasion and metastasis has become necessary for the treatment of GC.

The homeobox (HOX) proteins are transcription factors with roles in development, including regulating the patterning during embryogenesis and the control of cell differentiation $(5,6)$. In mammals, the HOX genes are organized into clusters named A, B, C and D on four separate chromosomes (7). The HOXA cluster contains 12 genes (11 HOX genes and EVX1) and is located in a $155 \mathrm{~kb}$-long genomic region on chromosome 7p15-7p14.2 (8). HOXA9 is normally expressed during development of the female reproductive tract, and its expression is tightly regulated in the adult tract $(9,10)$.

A previous study revealed that deregulated expression of HOX genes is found in cancers (11). However, another study demonstrated that HOX proteins function in a context-dependent manner (5). HOXA9 was revealed to exert a tumor-suppressive effect in breast cancer, reported by Gilbert et al (12). Uchida et al also demonstrated that HOXA9 acts as a tumor suppressor in oral cancer (13). Furthermore, methylation and loss of expression of HOXA9 was reported 
in oral cavity (14), breast $(12,15,16)$, lung (17), ovarian (18) and bladder (19) cancers.

In contrast to the tumor suppressor role, several studies have considered the oncogenic role of HOXA9 in human cancer $(11,20)$. Ko et al $(20)$ identified that high expression of HOXA9 is associated with poor overall survival (OS) in ovarian cancer, and HOXA9 could promote ovarian tumor growth in vivo. In addition, a previous study also demonstrated that HOXA9 may act as an oncogene in leukemia (11). Therefore, HOXA9 appears to exert its function by interacting with different types of proteins in a tissue-specific manner. However, the role of HOXA9 in gastric cancer is poorly understood.

PBX3 is a member of the PBX family of three-amino acid loop extension HOX genes. PBX proteins are well known for their interaction with HOX proteins that increases the DNA-binding affinity of HOX proteins, thereby enhancing the transcription of the downstream target genes $(11,21)$. A study by Li et al indicated that HOXA/PBX3 interaction is critical for mixed lineage leukemia-induced leukemia (22). The identification of this HOXA/PBX3 gene signature triggered the present study to investigate whether a synergistic effect exists between HOXA9 and PBX3 in GC. The aim of the present study was to evaluate the clinical significance of HOXA9 and PBX3 in the progression and prognosis of GC, and to explore the potential association between HOXA9 and $\mathrm{PBX} 3$ in GC progression.

\section{Materials and methods}

Patients and tissue samples. The project was approved by the ethics committee on the use of human subjects of Zhejiang Provincial People's Hospital (ZPPH; Hangzhou, China) and written informed consent was obtained from each patient. A total of 24 fresh specimens from patients with GC were acquired from ZPPH between January 2013 and December 2013 , and stored at $-80^{\circ} \mathrm{C}$ prior to use. Surrounding normal gastric mucosa samples were also obtained and studied.

In addition, 128 paraffin-embedded specimens of GC were collected at ZPPH between January 2006 and December 2009. All cases were diagnosed clinically at the Department of Gastrointestinal Surgery, and histopathologically at the Department of Pathology of ZPPH. The patient cohort consisted of 104 males and 24 females (Table I), with a median age of 54 years (range, 17-87 years) at the time of surgery.

All cases were classified according to the World Health Organization pathological classification of tumors. Among the 128 cases of GC, 16 were well differentiated, 51 were moderately differentiated and 61 were poorly differentiated. There were 62 cases without lymph node metastasis, 66 cases with lymph node metastasis, 2 cases with distant metastasis and 126 cases without distant metastasis. According to TNM stage classification, 61 cases were categorized as stage I+II and 67 cases were categorized as stage III+IV. None of the patients had received any radiotherapy or chemotherapy prior to surgery.

All patients were followed for $>5$ years, and the survival time was calculated from the date of surgery to the deadline for follow-up, or to the date of mortality.
Reverse transcription polymerase chain reaction (RT-PCR). Total RNA was extracted from the fresh specimens using TRIzol (Invitrogen; Thermo Fisher Scientific, Inc., Waltham, MA, USA) and RNA concentration was determined using a Nanodrop 2000 spectrophotometer (Thermo Fisher Scientific, Inc.). A total of $2 \mu \mathrm{g}$ of RNA was reverse transcribed using the SuperScript II reverse transcriptase system (Invitrogen; Thermo Fisher Scientific, Inc.) following the manufacturer's protocol. The cDNA was then subjected to RT-PCR using specific primers with the SYBR Premix ExTaq kit (Takara Bio, Inc., Otsu, Japan). The forward and reverse primers for HOXA9 (NM_152739) were 5'-GTGATG CCATTTGGGCTTATT-3' and 5'-GGTTTAGAGCCGCTT TGTGC-3', respectively. Those for PBX3 (NM_001134778) were 5'-CTG TTTGCCTATCCCTGTT-3' (forward) and 5'-GCAGCA AGTATCTTCGTCTC-3' (reverse). GAPDH was used as an internal control using the following primers: Forward, 5'-TGA AGGTCGGAGTCAACGG-3' and reverse, 5'-CTGGAA GATGGTGATGGGATT-3'. The relative amount of mRNA level to GAPDH was calculated as the average $2^{-\Delta \Delta \mathrm{Cq}}$, where $\Delta \mathrm{Cq}=\mathrm{Cq}-\mathrm{Cq}_{\mathrm{GAPDH}}(23)$.

Immunohistochemical staining. Each tissue section was baked at $60^{\circ} \mathrm{C}$ for $2 \mathrm{~h}$, deparaffinized with xylene and rehydrated in graded alcohol. Antigen retrieval was then performed by autoclaving in $0.01 \mathrm{M}$ citrate buffer ( $\mathrm{pH}$ 6.0) for $3 \mathrm{~min}$. Subsequently, sections were incubated with $3 \%(\mathrm{v} / \mathrm{v})$ $\mathrm{H}_{2} \mathrm{O}_{2}$ for 10 min to block endogenous peroxidase.

To reduce nonspecific reactions, sections were then incubated with $10 \%$ (vol/vol) normal goat serum (Histostain-Plus kit; cat. no. 859043; Invitrogen; Thermo Fisher Scientific, Inc.) for $15 \mathrm{~min}$ at room temperature. Subsequently, the slides were incubated overnight at $4{ }^{\circ} \mathrm{C}$ with rabbit polyclonal antibody against human HOXA9 (dilution, 1:500; cat. no. bs6667R; BIOSS, Beijing, China) or rabbit polyclonal antibody to human PBX3 (dilution, 1:500; cat. no. bs12295R; BIOSS). Subsequent to rinsing with PBS, tissue sections were incubated for $20 \mathrm{~min}$ at room temperature with biotin-labeled secondary antibody (Histostain-Plus kit; cat. no. 859043; Invitrogen; Thermo Fisher Scientific, Inc.) followed by horseradish peroxidase-linked goat anti-rabbit antibody (Histostain-Plus kit; cat. no. 859043; Invitrogen; Thermo Fisher Scientific, Inc.) for $20 \mathrm{~min}$ at room temperature. Sections were then stained with 3,3-diaminobenzidine (ZSGB-BIO, Beijing, China). Finally, the sections were counterstained with hematoxylin (Sigma-Aldrich; Merck KGaA, Darmstadt, Germany), dehydrated and mounted with a coverslip. Phosphate buffer was used to replace the primary antibody as a negative control.

Evaluation of immunohistochemical staining. Immunohistochemical staining showed that HOXA9 and PBX3 positive staining were mainly located in the nucleus and cytoplasm. The degree of immunostaining was reviewed under a light microscope (5 fields were viewed with magnification x200) by two expert pathologists without knowledge of the clinical data and scored independently. The HOXA9 and PBX3 expression level was based on the intensity of cellular staining and the proportion of stained tumor cells.

Staining intensity was scored according to the following criteria: 0 , no staining; 1, weak staining (light yellow); 
Table I. Association of HOXA9 and PBX3 expression with clinicopathological features of patients with gastric cancer.

\begin{tabular}{|c|c|c|c|c|c|c|c|}
\hline \multirow[b]{2}{*}{ Clinicopathological features } & \multirow[b]{2}{*}{ Total } & \multicolumn{3}{|c|}{ Positive HOXA9 expression } & \multicolumn{3}{|c|}{ Positive PBX3 expression } \\
\hline & & Patients, n (\%) & $\chi^{2}$ & P-value & Patients, n (\%) & $\chi^{2}$ & P-value \\
\hline Gender & & & 0.060 & 0.807 & & 0.777 & 0.378 \\
\hline Male & 104 & $72(69.2)$ & & & $73(70.2)$ & & \\
\hline Female & 24 & $16(66.7)$ & & & $19(79.2)$ & & \\
\hline Age range & & & 0.180 & 0.671 & & 0.020 & 0.887 \\
\hline$<60$ years & 83 & $56(67.5)$ & & & $60(72.3)$ & & \\
\hline$\geq 60$ years & 45 & $32(71.1)$ & & & $32(71.1)$ & & \\
\hline Differentiation & & & 14.896 & 0.001 & & 1.890 & 0.420 \\
\hline Well & 16 & $8(50.0)$ & & & $10(62.5)$ & & \\
\hline Moderate & 51 & $28(54.9)$ & & & $35(68.6)$ & & \\
\hline Poor & 61 & $52(85.2)$ & & & $47(77.0)$ & & \\
\hline Lymph node metastasis & & & 6.390 & 0.011 & & 17.264 & $<0.001$ \\
\hline Negative & 62 & $36(58.1)$ & & & $34(54.8)$ & & \\
\hline Positive & 66 & $52(78.8)$ & & & $58(87.9)$ & & \\
\hline Distant metastasis & & & 0.924 & 1.000 & & 0.795 & 1.000 \\
\hline Negative & 126 & $86(68.3)$ & & & $90(71.4)$ & & \\
\hline Positive & 2 & $2(100.0)$ & & & $2(100.0)$ & & \\
\hline TNM stage & & & 14.396 & 0.0001 & & 29.692 & $<0.001$ \\
\hline $\mathrm{I}+\mathrm{II}$ & 61 & $32(52.5)$ & & & $30(49.2)$ & & \\
\hline $\mathrm{III}+\mathrm{IV}$ & 67 & $56(83.6)$ & & & $62(92.5)$ & & \\
\hline
\end{tabular}

HOXA9, homeobox A9; PBX3, PBX homeobox 3; TNM, tumor-node-metastasis.

2, moderate staining (yellow brown); and 3, strong staining (brown). The proportion of stained tumor cells was scored according to the proportion of positively stained tumor cells, as follows: $0,<5 \%$ positive tumor cells; $1,6-25 \%$ positive tumor cells; $2,26-50 \%$ positive tumor cells; and $3,>51 \%$ positive tumor cells. The staining intensity and proportion immunoreactivity scores were then multiplied to obtain a composite score. The values of the composite score ranged from 0 to 9 . For additional evaluation, a staining index score of $\leq 4$ was defined as HOXA9 or PBX3 negative expression, and a staining index score of $>5$ was regarded as HOXA9 orPBX3 positive expression. In cases of discrepancy, a consensus score was chosen for evaluation.

Statistical analysis. All statistical analyses were performed using SPSS 13.0 software (SPSS, Inc., Chicago, IL, USA). Differences between HOXA9 and PBX3 mRNA expression levels of cancer and normal tissues were determined using the Mann-Whitney U test. $\chi^{2}$ test or Fisher's exact test was used to evaluate the associations between the expression of HOXA9 or PBX3 and the clinicopathological features of the patients with GC. Univariate survival analysis was performed using the Kaplan-Meier method, accompanying the log-rank test to calculate differences among the curves. Multivariate survival analysis was performed to assess predictors associated with prognosis using Cox proportional hazards regression model. Additionally, association between HOXA9 expression, PBX3 expression and clinicopathological features was estimated using Spearman's rank correlation coefficient. All P-values were two-sided and $\mathrm{P}<0.05$ was considered to indicate a statistically significant difference.

\section{Results}

Detection of HOXA9 and PBX3 mRNA expression level. To detect HOXA9 and PBX3 mRNA expression level, a total of 24 paired fresh specimens of GC and surrounding normal mucosa were analyzed using RT-PCR. The results revealed that the HOXA9 mRNA level was upregulated in $62.5 \%$ of GCs $(15 / 24)$, and downregulated in $37.5 \%$ of the GCs (9/24), and the mean mRNA level of HOXA9 was upregulated in GC tissues compared with normal tissues $(\mathrm{P}=0.032$; Fig. 1).

Similarly, PBX3 was significantly upregulated in $79.2 \%$ of GCs $(19 / 24)$ and downregulated in the remainder of GCs $(5 / 24,20.8 \%)$, and the mean mRNA level of PBX3 was upregulated in GC tissues compared with normal tissues ( $\mathrm{P}=0.031$; Fig. 1).

Further analysis of the association between HOXA9 and PBX3 mRNA level was also performed, and the result showed that the HOXA9 mRNA level was significantly correlated with PBX3 mRNA level ( $r=0.358 ; \mathrm{P}=0.012$; Fig. 2$)$.

Association between HOXA9 and PBX3 expression with clinicopathological features of $G C$. In order to detect the presence and distribution of HOXA9 and PBX3 expression 
A

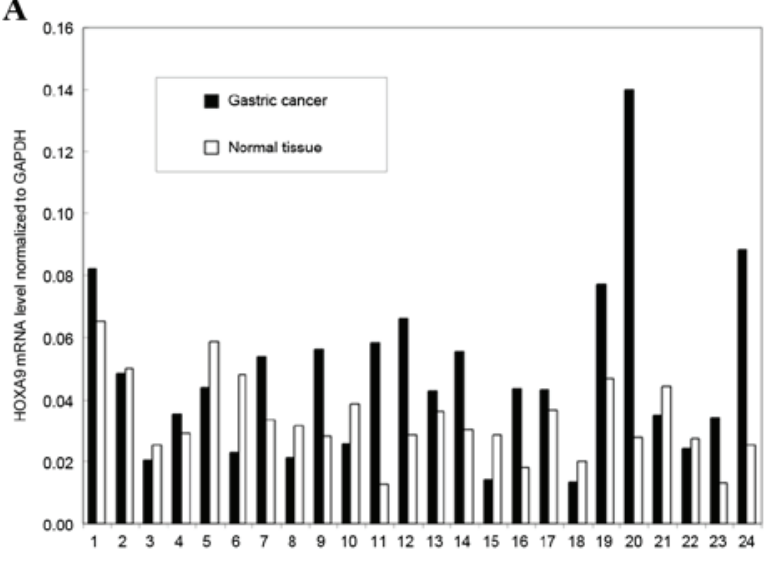

C

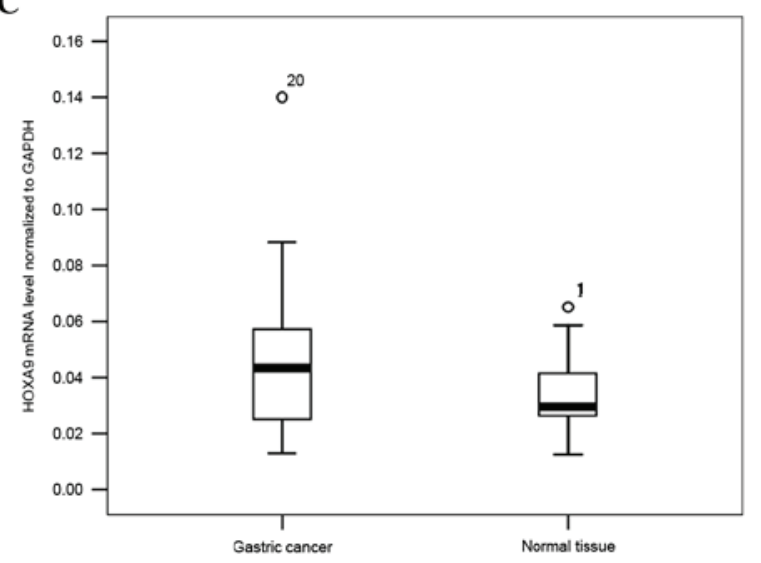

B

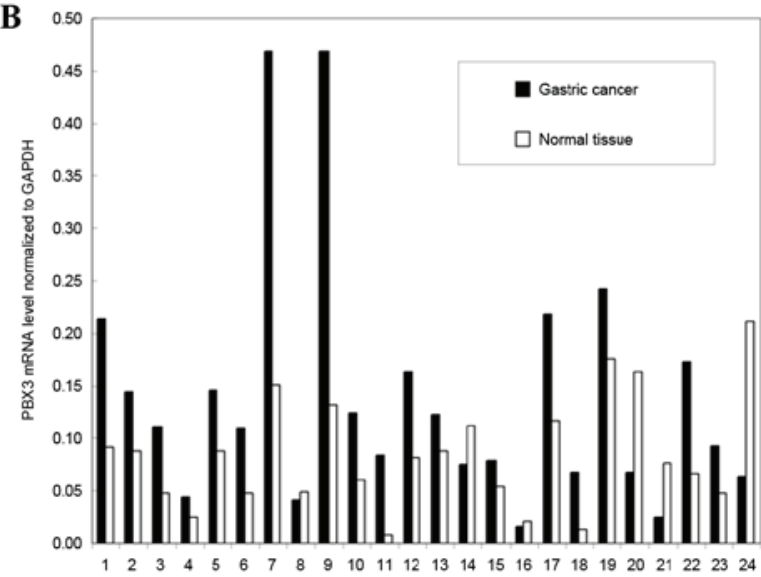

D

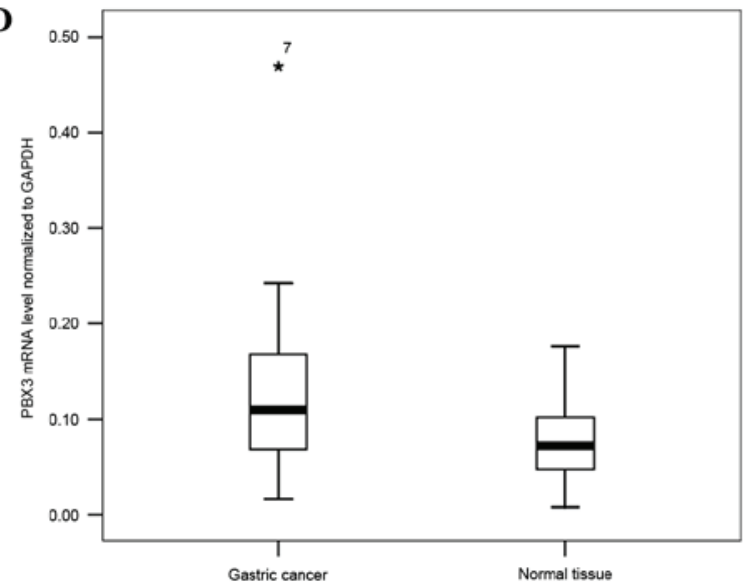

Figure 1. Gene expression of HOXA9 and PBX3 in GC and adjacent normal tissue. (A) The relative mRNA levels of HOXA9 normalized to GAPDH in the 24 paired specimens. (B) The relative mRNA levels of PBX3 normalized to GAPDH in the 24 paired specimens. (C) The mean expression levels of HOXA9 mRNA in GC and in normal tissue ( $\mathrm{P}=0.032$ ). (D) The mean expression levels of PBX3 mRNA in GC and in normal tissue ( $\mathrm{P}=0.031$ ). GC, gastric cancer; HOXA9, homeobox A9; PBX3, PBX homeobox 3. ${ }^{\circ}$ represents discrete numeric value; ${ }^{*}$ represents extreme values.

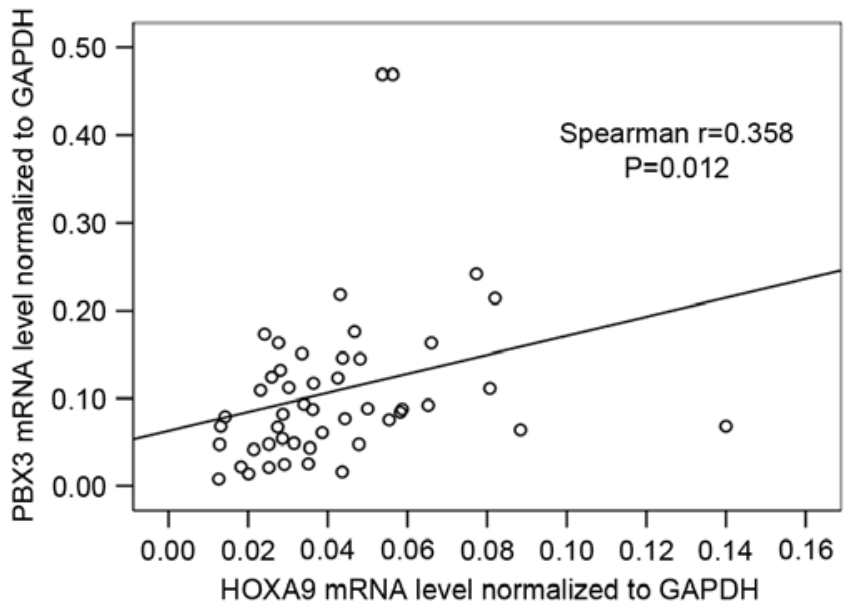

Figure 2. Spearman's rank correlation analysis of HOXA9 and PBX3 mRNA expression. The $\mathrm{x}$-axis shows HOXA9 mRNA level relative to GAPDH, while the $\mathrm{y}$-axis shows PBX3 mRNA level relative to GAPDH. $r=0.358 ; \mathrm{P}=0.012$. HOXA9, homeobox A9; PBX3, PBX homeobox 3.

in GC, immunohistochemical staining was performed, and the association between HOXA9 and PBX3 expression with clinicopathological features of GC was analyzed. The results revealed that immunostaining of HOXA9 was mainly located in the nucleus and cytoplasm of the tumor cells (Fig. 3), and positive expression of HOXA9 was detected in 88 of the 128 patients with GC $(68.8 \%)$. Additional analysis demonstrated that HOXA9 expression was associated with differentiation, lymph node metastasis and TNM stage (Table I). Gastric cancer patients with poor differentiation, lymph node metastasis and high TNM stage (stages III+IV) had significantly increased expression of HOXA9 compared with those with well or moderate differentiation $(\mathrm{P}=0.001)$, no lymph node metastasis $(\mathrm{P}=0.011)$ and low TNM stage (stages I+II) (P=0.0001; Table I). The Spearman's rank correlation coefficient of HOXA9 expression with differentiation, lymph node metastasis and TNM stage was $0.185(\mathrm{P}=0.037)$, $0.298(\mathrm{P}=0.001)$ and $0.439(\mathrm{P}<0.001)$, respectively.

Immunostaining of PBX3 was predominantly distributed in the nucleus and cytoplasm of the tumor cells (Fig. 3), and positive expression of PBX3 was detected in 92 of the 128 patients with GC (71.9\%). PBX3 expression was associated with lymph node metastasis and TNM stage (Table I). Positive expression of PBX3 was detected in $87.9 \%$ (58/66) of GC patients with lymph node metastasis, which was increased compared with the expression rate in patients without lymph node metastasis $(34 / 62,54.8 \%)\left(\chi^{2}=17.264 ; \mathrm{P}<0.001\right)$. The detection rate of PBX3 expression was $92.5 \%$ (62/67) in GC patients with TNM stage III+IV, which revealed a significant 


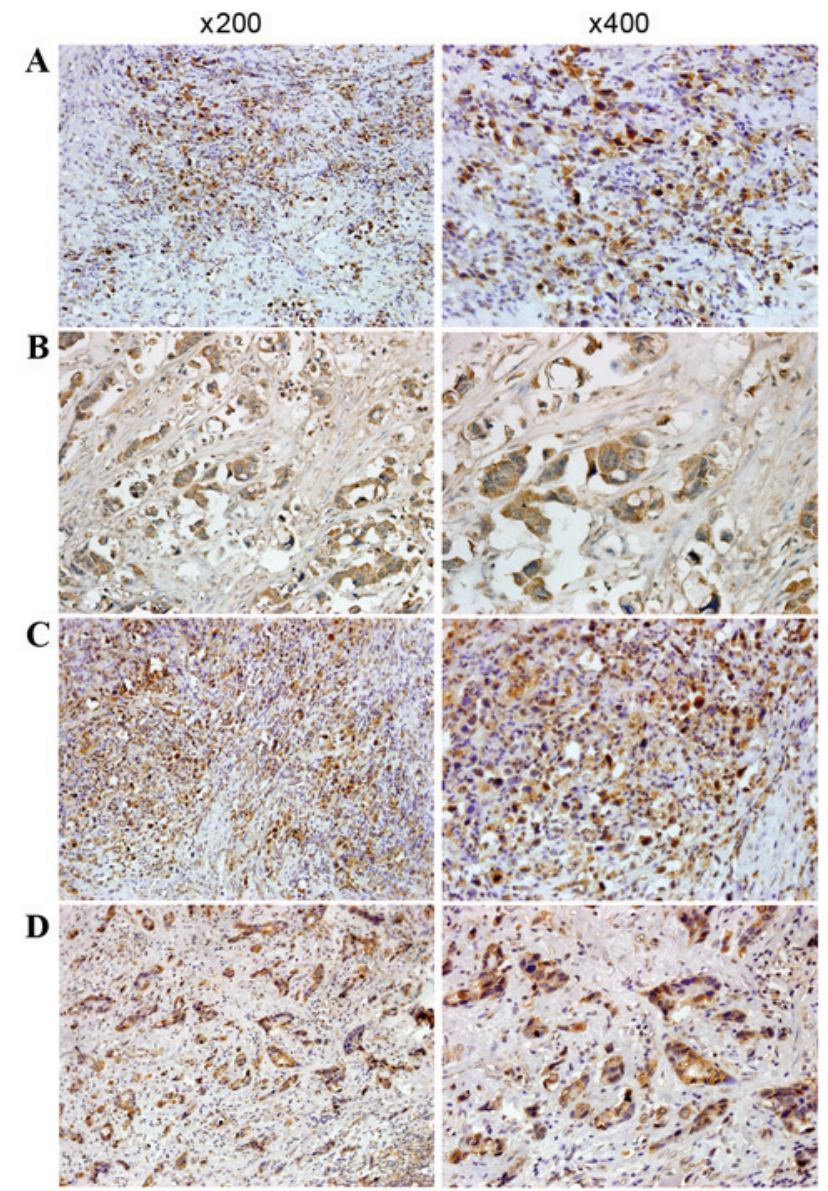

Figure 3. Immunohistochemical staining for HOXA9 and PBX3 in GC and in normal tissue. (A) Immunostaining of HOXA9 in poorly-differentiated GC tissue revealed that positive staining was mainly in the nucleus and also in the cytoplasm. (B) Immunostaining of HOXA9 in moderately-differentiated GC tissue. Positive staining was mainly in the nucleus and cytoplasm. (C) Immunostaining of PBX3 in poorly-differentiated GC tissue. Positive staining was mainly in the nucleus and also in the cytoplasm. (D) Immunostaining of PBX3 in moderately differentiated GC tissue and positive staining was mainly in the nucleus and cytoplasm. GC, gastric cancer; HOXA9, homeobox A9; PBX3, PBX homeobox 3. Magnification, x200 (left) and x400 (right).

difference from TNM stage I+II $\left(49.2 \% ; \chi^{2}=26.692 ; \mathrm{P}<0.001\right)$. Furthermore, the Spearman's rank correlation coefficients of PBX3 expression with lymph node metastasis and TNM stage were $0.438(\mathrm{P}<0.001)$ and $0.579(\mathrm{P}<0.001)$, respectively.

Association between expression of HOXA9 and PBX3 in GC. In order to investigate the synergistic effect between HOXA9 and PBX3 in GC, the association between HOXA9 and PBX3 in the development of gastric cancer was analyzed. High coincidental expression of the HOXA9 and PBX3 proteins was observed in gastric cancer. Of the 88 patients that were found to express HOXA9, 69 (78.4\%) also expressed PBX3. The correlation between the expression of HOXA9 and PBX3 expression in patients with gastric cancer was statistically significant $(r=0.391 ; \mathrm{P}<0.001)$.

Clinical significance of HOXA9 and PBX3 expression in prognosis of $G C$. Univariate survival analysis revealed the 3 - and 5-year cumulative survival rates were 81.6 and
47.8\% in patients with negative HOXA9 expression, and 56.3 and $14.1 \%$ in those with positive HOXA9 expression. The mean survival time in patients of GC with positive HOXA9 expression was $37.96 \pm 2.22$ months, and 50.69 \pm 3.02 months for those with negative HOXA9 expression. Evidently, GC patients with positive expression of HOXA9 have a poorer prognosis than those with negative expression $\left(\chi^{2}=7.378\right.$; $\mathrm{P}=0.007$; Fig. 4).

Similarly, the 3- and 5-year cumulative survival rates were 79.8 and $62.1 \%$ in patients with negative expression of PBX3, which were increased compared with patients with positive expression of PBX3 (58.4 and 11.2\%, respectively). The mean survival time in patients of GC with positive expression of

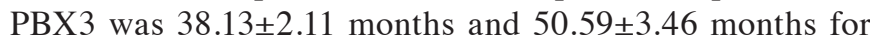
those with negative expression of PBX3. Notably, GC patients with positive expression of $\mathrm{PBX} 3$ had a poorer prognosis than those with negative expression $\left(\chi^{2}=9.948 ; \mathrm{P}=0.002\right.$; Fig. 4). Multivariate analysis using the Cox regression model demonstrated that survival was independently associated with lymph node metastasis $(\mathrm{P}=0.032)$ and $\mathrm{PBX} 3$ expression $(\mathrm{P}=0.024$; Table II $)$.

\section{Discussion}

HOX genes are an important class of patterning regulators that modulate tumor progression and alter tumor cell growth in vitro (24-26). HOX proteins can form heterodimers or heterotrimers with members of the 3-amino-acid loop extension family of cofactors, including PBX and Meis proteins, which may directly regulate the transcription of downstream target genes. Previous studies showed that HOXA9 appears to exert its function by interacting with different types of proteins in a tissue-specific manner $(5,11,12,20)$. However, the role of HOXA9 in gastric cancer has not been fully elucidated.

To understand the clinicopathological significance of HOXA9 in GC, the expression of HOXA9 mRNA level was analyzed in 34 paired fresh GC tissue and 128 paraffin-embedded GC tissues. In the present study, HOXA9 and PBX3 mRNA levels were revealed to be significantly upregulated in GC tissue compared with adjacent normal tissue. Immunohistochemical staining also revealed that gastric cancer patients with poor differentiation, lymph node metastasis and high TNM stage (stages III+IV) had significantly increased expression of HOXA9 compared with patients with well or moderate differentiation $(\mathrm{P}=0.001)$, no lymph node metastasis $(\mathrm{P}=0.011)$ and low TNM stage (stages I+II; $\mathrm{P}=0.0001$ ). These results showed that HOXA9 overexpression was involved in the progression of GC. HOXA9 has been implicated in carcinogenesis, since it acts as a transcription factor with roles in development, regulating patterning during embryogenesis and controlling cell differentiation $(5,6)$. Studies also revealed that HOXA9 increases endothelial cell migration and tube formation in human myeloid leukemia cells $(27,28)$. In addition, HOXA9 promotes tumor metastasis by enhancing the adhesion of circulating tumor cells to endothelial cells (29). HOXA9 was also reported to increase cell proliferation and inhibit apoptosis in human glioblastoma (30). These findings indicate that HOXA9 may be involved in tumor progression by 
Table II. Multivariate analysis of the correlation between clinicopathological parameters and prognosis in patients with gastric cancer.

\begin{tabular}{lccccc}
\hline Covariates & Coefficient & Standard error & HR & 95\% CI & P-value \\
\hline Gender & -0.073 & 0.340 & 0.929 & $0.477-1.810$ & 0.829 \\
Age & 0.001 & 0.299 & 1.001 & $0.557-1.799$ & 0.998 \\
Differentiation & -0.192 & 0.189 & 0.825 & $0.570-1.196$ & 0.310 \\
TNM stage & -1.048 & 0.616 & 0.350 & $0.105-1.171$ & 0.089 \\
Lymph node metastasis & 1.166 & 0.542 & 3.209 & $1.109-9.287$ & 0.032 \\
Distant metastasis & 0.741 & 0.794 & 2.097 & $0.442-9.942$ & 0.351 \\
HOXA9 expression & 0.637 & 0.351 & 1.890 & $0.951-3.758$ & 0.069 \\
PBX3 expression & 0.885 & 0.393 & 2.424 & $1.123-5.233$ & 0.024 \\
\hline
\end{tabular}

HR, hazard ratio; CI, confidence interval; HOXA9, homeobox A9; PBX3, PBX homeobox 3; TNM, tumor-node-metastasis.

A

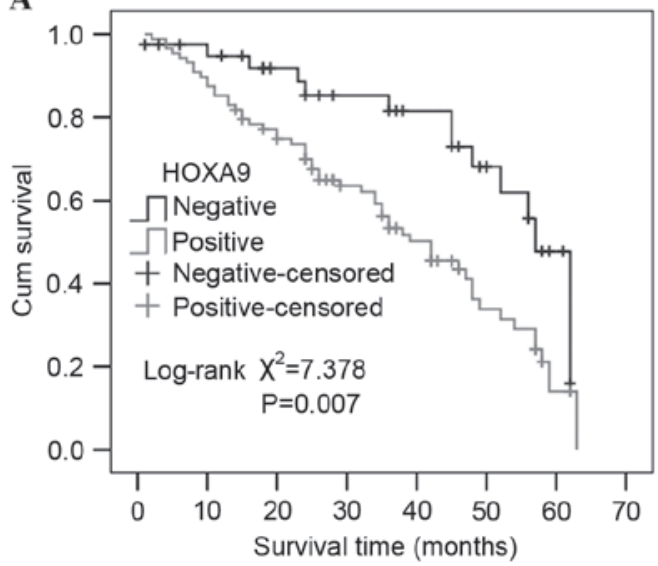

B

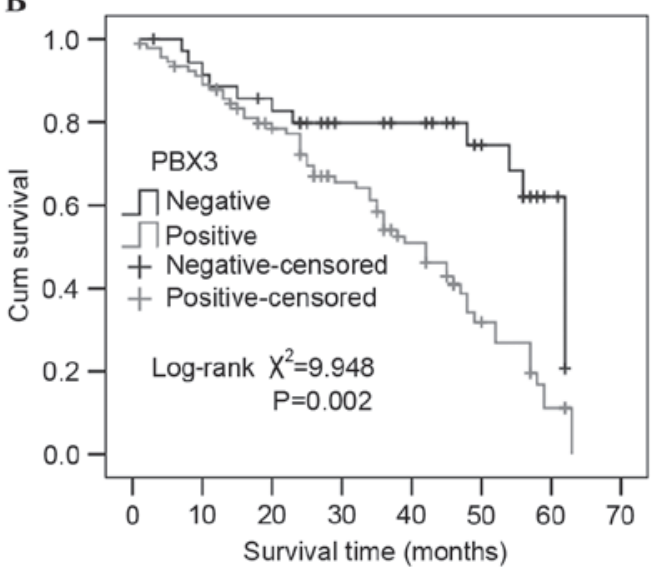

Figure 4. Kaplan-Meier survival curve analysis in patients with HOXA9 or PBX3 expression. The cumulative survival rate of patients with positive expression of (A) HOXA9 or (B) PBX3 was significantly decreased compared with the survival rate of patients with negative expression $(\mathrm{P}=0.007$ and $\mathrm{P}=0.002$, respectively). HOXA9, homeobox A9; PBX3, PBX homeobox 3.

modulating interactions between tumor cells and host cells. However, a number of studies revealed that HOXA9 exerted a tumor-suppressive effect in breast cancer, lung cancer, ovarian cancer and bladder cancer (12,17-19). HOXA9 is frequently deregulated in a variety of human cancers, in which it acts as a tumor suppressor or as an oncogene. Although HOXA9 seems to exert its function by interacting with different types of proteins in a tissue-specific manner, the mechanisms underlying these differential functions remain to be identified.

PBX proteins are also well known for their interaction with HOX proteins, which increase the DNA-binding affinity of HOX proteins and thereby enhance the transcription of the downstream target genes $(11,31,32)$. The cooperation between PBX3 proteins and HOXA9 in GC progression is unclear. The present study revealed hat HOXA9 mRNA level was associated with that of PBX3. Immunohistochemical staining also revealed a high coincidental expression of the HOXA9 and $\mathrm{PBX} 3$ proteins in GC. Additional analysis revealed hat PBX3 expression was associated with lymph node metastasis and TNM stage. Positive expression rates of PBX3 were increased in GC patients with lymph node metastasis and
TNM stage III+IV compared with patients without lymph node metastasis and TNM stage I+II. Therefore, the present data suggest that PBX3 may be a critical cofactor of HOXA9 in GC carcinogenesis and development.

Survival analysis also revealed that high expression of HOXA9 or PBX3 was associated with poor survival of GC, and multivariate analysis using the Cox regression model showed that PBX3 expression was an independent prognostic factor in GC. High HOXA9 expression was reported to be associated with poor OS of epithelial ovarian carcinoma patients (20). Li et al also demonstrated that increased expression of a 4-HOX gene signature (composed of HOXA7, HOXA9, HOXA11 and PBX3) is an independent predictor of shortened OS in patients with cytogenetically abnormal acute myeloid leukemia (33). The present study showed that the HOXA9/PBX3 gene signature has a prognostic value for GC.

On the basis of these findings, it is suggested that PBX3 is a critical cofactor of HOXA9, and that cross-talk between HOXA9 and PBX3 may perform an important role in the mechanism underlying the carcinogenesis, development and progression of GC. Therefore, targeting the interaction of these 
genes is a feasible strategy for the therapy of GC; however, the mechanisms underlying the regulation of HOXA9/PBX3 in $\mathrm{GC}$ development remain to be identified.

\section{Acknowledgements}

The present study was supported by the Natural Science Foundation of Zhejiang Province (grant no. LQ16H160017 to Ying-Yu Ma and grant no. LY15H160051 to Xiao-Zhou Mou).

\section{References}

1. Ferlay J, Shin HR, Bray F, Forman D, Mathers C and Parkin DM: Estimates of worldwide burden of cancer in 2008: GLOBOCAN 2008. Int J Cancer 127: 2893-2917, 2010.

2. Mullen JT and Ryan DP: Neoadjuvant chemotherapy for gastric cancer: What are we trying to accomplish? Ann Surg Oncol 21: $13-15,2014$.

3. Waddell T, Verheij M, Allum W, Cunningham D, Cervantes A and Arnold D; European Society for Medical Oncology (ESMO); European Society of Surgical Oncology (ESSO); European Society of Radiotherapy and Oncology (ESTRO): Gastric cancer: ESMO-ESSO-ESTRO clinical practice guidelines for diagnosis, treatment and follow-up. Eur J Surg Oncol 40: 584-591, 2014

4. Bogenrieder T and Herlyn M: Axis of evil: Molecular mechanisms of cancer metastasis. Oncogene 22: 6524-6536, 2003

5. Pearson JC, Lemons D and McGinnis W: Modulating Hox gene functions during animal body patterning. Nat Rev Genet 6 : 893-904, 2005

6. Samuel S and Naora H: Homeobox gene expression in cancer: Insights from developmental regulation and deregulation. Eur J Cancer 41: 2428-2437, 2005.

7. Krumlauf R: Hox genes in vertebrate development. Cell 78 : 191-201, 1994

8. Rauch T, Wang Z, Zhang X, Zhong X, Wu X, Lau SK, Kernstine KH, Riggs AD and Pfeifer GP: Homeobox gene methylation in lung cancer studied by genome-wide analysis with a microarray-based methylated $\mathrm{CpG}$ island recovery assay. Proc Natl Acad Sci USA 104: 5527-5532, 2007.

9. Cheng W, Liu J, Yoshida H, Rosen D and Naora H: Lineage infidelity of epithelial ovarian cancers is controlled by HOX genes that specify regional identity in the reproductive tract. Nat Med 11: 531-537, 2005.

10. Vitiello D, Kodaman PH and Taylor HS: HOX genes in implantation. Semin Reprod Med 25: 431-436, 2007.

11. Shah N and Sukumar S: The Hox genes and their roles in oncogenesis. Nat Rev Cancer 10: 361-371, 2010.

12. Gilbert PM, Mouw JK, Unger MA, Lakins JN, Gbegnon MK, Clemmer VB, Benezra M, Licht JD, Boudreau NJ, Tsai KK, et al: HOXA9 regulates BRCA1 expression to modulate human breast tumor phenotype. J Clin Invest 120: 1535-1550, 2010.

13. Uchida K, Veeramachaneni R, Huey B, Bhattacharya A, Schmidt BL and Albertson DG: Investigation of HOXA9 promoter methylation as a biomarker to distinguish oral cancer patients at low risk of neck metastasis. BMC Cancer 14: 353 , 2014.

14. Guerrero-Preston R, Soudry E, Acero J, Orera M, Moreno-López L, Macía-Colón G, Jaffe A, Berdasco M, Ili-Gangas C, Brebi-Mieville P, et al: NID2 and HOXA9 promoter hypermethylation as biomarkers for prevention and early detection in oral cavity squamous cell carcinoma tissues and saliva. Cancer Prev Res (Phila) 4: 1061-1072, 2011.

15. Reynolds PA, Sigaroudinia M,Zardo G, Wilson MB, Benton GM, Miller CJ, Hong C, Fridlyand J, Costello JF and Tlsty TD: Tumor suppressor p16INK4A regulates polycomb-mediated DNA hypermethylation in human mammary epithelial cells. J Biol Chem 281: 24790-24802, 2006.

16. Sun M, Song CX, Huang H, Frankenberger CA, Sankarasharma D, Gomes S, Chen P, Chen J, Chada KK, He C and Rosner MR: HMGA2/TET1/HOXA9 signaling pathway regulates breast cancer growth and metastasis. Proc Natl Acad Sci USA 110: 9920-9925, 2013.
17. Son JW, Jeong KJ, Jean WS, Park SY, Jheon S, Cho HM, Park CG, Lee HY and Kang J: Genome-wide combination profiling of DNA copy number and methylation for deciphering biomarkers in non-small cell lung cancer patients. Cancer Lett 311: 29-37, 2011.

18. Wu Q, Lothe RA, Ahlquist T, Silins I, Tropé CG, Micci F, Nesland JM, Suo Z and Lind GE: DNA methylation profiling of ovarian carcinomas and their in vitro models identifies HOXA9, HOXB5, SCGB3A1, and CRABP1 as novel targets. Mol Cancer 6: 45, 2007.

19. Reinert T, Borre M, Christiansen A, Hermann GG, Ørntoft TF and Dyrskjot L: Diagnosis of bladder cancer recurrence based on urinary levels of EOMES, HOXA9, POU4F2, TWIST1, VIM and ZNF154 hypermethylation. PLoS One 7: e46297, 2012.

20. Ko SY, Barengo N, Ladanyi A, Lee JS, Marini F, Lengyel E and Naora H: HOXA9 promotes ovarian cancer growth by stimulating cancer-associated fibroblasts. J Clin Invest 122: 3603-3617, 2012

21. Chang CP, Brocchieri L, Shen WF, Largman C and Cleary ML: $\mathrm{Pbx}$ modulation of Hox homeodomain amino-terminal arms establishes different DNA-binding specificities across the Hox locus. Mol Cell Biol 16: 1734-1745, 1996.

22. Li Z, Zhang Z, Li Y, Arnovitz S, Chen P, Huang H, Jiang X, Hong GM, Kunjamma RB, Ren H, et al: PBX3 is an important cofactor of HOXA9 in leukemogenesis. Blood 121: 1422-1431, 2013.

23. Livak KJ and Schmittgen TD: Analysis of relative gene expression data using real-time quantitative PCR and the 2(-Delta Delta C(T)) Method. Methods 25: 402-408, 2001.

24. Muratovska A, Zhou C, He S, Goodyer P and Eccles MR: Paired-Box genes are frequently expressed in cancer and often required for cancer cell survival. Oncogene 22: 7989-7997, 2003.

25. Tan Y, Cheung M, Pei J, Menges CW, Godwin AK and Testa JR: Upregulation of DLX5 promotes ovarian cancer cell proliferation by enhancing IRS-2-AKT signaling. Cancer Res 70: 9197-9206, 2010.

26. Trinh BQ, Ko SY, Barengo N, Lin SY and Naora H: Dual functions of the homeoprotein DLX4 in modulating responsiveness of tumor cells to topoisomerase II-targeting drugs. Cancer Res 73: 1000-1010, 2013.

27. Bruhl T, Urbich C, Aicher D, Acker-Palmer A, Zeiher AM and Dimmeler S: Homeobox A9 transcriptionally regulates the EphB4 receptor to modulate endothelial cell migration and tube formation. Circ Res 94: 743-751, 2004

28. Nakamura T, Largaespada DA, Lee MP, Johnson LA, Ohyashiki K, Toyama K, Chen SJ, Willman CL, Chen IM, Feinberg AP, et al: Fusion of the nucleoporin gene NUP98 to HOXA9 by the chromosome translocation $\mathrm{t}(7 ; 11)(\mathrm{p} 15 ; \mathrm{p} 15)$ in human myeloid leukaemia. Nat Genet 12: 154-158, 1996.

29. Bandyopadhyay S, Ashraf MZ, Daher P, Howe PH and DiCorleto PE: HOXA9 participates in the transcriptional activation of E-selectin in endothelial cells. Mol Cell Biol 27: 4207-4216, 2007

30. Costa BM, Smith JS, Chen Y, Chen J, Phillips HS, Aldape KD, Zardo G, Nigro J, James CD, Fridlyand J, et al: Reversing HOXA9 oncogene activation by PI3K inhibition: Epigenetic mechanism and prognostic significance in human glioblastoma. Cancer Res 70: 453-462, 2010.

31. Chang CP, Shen WF, Rozenfeld S, Lawrence HJ, Largman C and Cleary ML: Pbx proteins display hexapeptide-dependent cooperative DNA binding with a subset of Hox proteins. Genes Dev 9: 663-674, 1995.

32. Milech N, Kees UR and Watt PM: Novel alternative PBX3 isoforms in leukemia cells with distinct interaction specificities. Genes Chromosomes Cancer 32: 275-280, 2001.

33. Li Z, Huang H, Li Y, Jiang X, Chen P, Arnovitz S, Radmacher MD, Maharry K, Elkahloun A, Yang X, et al: Up-regulation of a HOXA-PBX3 homeobox-gene signature following down-regulation of miR-181 is associated with adverse prognosis in patients with cytogenetically abnormal AML. Blood 119: 2314-2324, 2012. 